\title{
Information Economic Man Assumption: Concept Definition and Comparison
}

\author{
Min Zhang ${ }^{1}$, Zhenggang $\mathrm{Chi}^{2} \&$ Jing Luo ${ }^{1}$ \\ ${ }^{1}$ Department of Economics and Management, Huizhou University, Huizhou, Guangdong, China \\ ${ }^{2}$ Department of Electronic Science, Huizhou University, Huizhou, Guangdong, China \\ Corresponding: Jing Luo, Department of Economics and Management, Huizhou University, Huizhou 516007, \\ Guangdong, China.
}

Received: November 9, 2017

Accepted: February 8, 2018

Online Published: March 8, 2018

doi:10.20849/abr.v3i1.333

URL: https://doi.org/10.20849/abr.v3i1.333

\begin{abstract}
Social norm, an important and coordinating part in economic activities, can greatly reduce the information demand that economic decisions require. The assumption of "Rational Man", which exists in the western mainstream economics, is based on the simplified model of the instinct of human beings. And this thoroughly ignores the social attribute of human beings. In the literature review, the assumption of "Rational Man" is analyzed as an abstract and deductive way based on the western culture of Christianity. This theory can better explain the economic behaviors of human beings under the specific social circumstance of the protestant ethic. However, its rationality cannot be guaranteed without such cultural background. Besides, under the eastern social circumstance dominated by Confucianism, the assumption of "Rational Man" can also bring prominent ethical conflicts. The assumption of "Information Economic Man" put forward by this thesis gives consideration both to the individual rationality and social features an economic man possesses, and better explains the impact of social norm on economic decisions. By bringing into the capital vector, and drawing on field theory, the model of "information field" is generated. It can provide economic man with a concise math model that affects the process of decision making. The size and direction of capital vector represent respectively the influence of economic factors and moral factors on economic decisions. And thus a wider range of economic behavior of human beings can be analyzed in a rational way.
\end{abstract}

Keywords: information economic man assumption, cooperation, information field, capital vector, social norm

\section{Introduction}

As the logical starting point of the basic behavioral hypothesis and theoretical system in modern western mainstream economic theory, the assumption of "Rational Man", is on one hand deemed by some scholars as the most effective method. "Compared with other patterns of human behaviors, the 'Economic Man' pattern, combined with the "Rational Behavior' assumption, may be the most pervasive, effective and successful method to explain human behaviors". (Shuguang Zhang, 1999) On the other hand, however, the assumption has been constantly doubted and criticized by many subjects of social science and even by the economic circles themselves.

Sub-disciplines of social science deal with the essence and the law of social things from various angles and dimensions. On the ground of their research targets, different sub-disciplines respectively put forward different hypothesis models over the behavior of human beings in social activities. These models, as abstract simplifications of real-world human behaviors in a specific dimension, should thus be coordinated and consistent with each other despite their differences. Nevertheless, over this issue, there is a deep gap between modern western mainstream economics and other disciplines. Herbert Gintis, an American scholar, points out that"Models of human interaction in economics highly contradict with those in sociology. Economics traditionally regards an individual as someone who is rational, self-regarding and maximizes his profit whereas sociology regards an individual as a highly socialized, other-regarding, moral behavior subject that plays his social roles and obtain his self-esteem from others'approval." (Herbert Gintis, 2011) Apparently, the assumption of rational economic man overemphasizes the importance of natural attributes of human beings and overlooks their social attributes. Besides, as the influence of economics is increasing in the area of social science, this fundamental assumption has generated considerable disputes and meanwhile, many other models of hypothesis 
of human behavior are set up. By summarizing "Hawthorne Experiment", Professor Mayo George Elton (1880-1949) of Harvard University proposed the assumption of "Social Man" in his book The Human Problem of an Industrial Civilization published in 1933. Mayo argued that to an individual, cooperation weighs more than competition and that the impetus of an individual action is not only to pursue individual interest but to guarantee the individual's status in an organization. The mind and action of a person is guided by his emotions more than by his rational logic. (Deqing Wang, 2004)

Abraham .H. Maslow (1908-1970), a behavioral scientist from America, proposed a theory of "Self-actualizing Man" in his book Motivation and Personality in 1954.(Xinjian Feng, 2001) According to the importance and the sequence of occurrence, Maslow sorted human needs into a pyramid shape. From low to high, successively there are physical need need of safety and security $\rightarrow$ need of belongingness and love $\rightarrow$ need of self-esteem $\rightarrow$ need of self-actualization, five needs in total. He also deemed that high-level needs could not be achieved unless low-level needs are fulfilled. Maslow argued that "a man's whole life is in endless pursuit and man is an animal that constantly needs something."(Frank Goble, 1987)

In 1957, in his representative book The Human Side of Enterprise, McGregor initially probed into the theory of human nature, a part of the theory of management, in a systematic way. He proposed "two assumptions of the view of human nature" and pointed out that "The nature and behavior of human are the most two important factors that determine the behavior pattern of an administrator. On the basis of their assumption of human nature, administrators accordingly organize, lead, control and stimulate people in various manners."(Daniel A., 2000)

Edgar Schein, a behavioral scientist from America, sorted out the matter of human nature and proposed the assumption of "Complex Man" in his book Organizational Psychology published in 1965. He argued that "human possesses complex motivations which should not be classified into one or two types simply. Besides, not all people are of the same type. As a matter of fact, there are various types of person."(Yaojun Sun, 1999)

Economic historian Karl Polanyi published his thesis Economy as process of system and proposed embedded theory. He pointed out that economic activities are embedded in specific social relation and social network. Thus, economic rationality based on the assumption of rational economic man should be embedded in a specific social circumstance. Only by this means can the assumption make a reasonable explanation for the reality. That is to say, "Economic Man" must be "Social Man". (Deshun Zang, 2009)

Likewise, scholars in China have done a large amount of research on this field. Jingtong He argued that behavioral man possesses dual motives of egoism as well as altruism that are two-dimensional orthogonal. On this basis, he proposed the assumption of "Realistic Economic Man". (Jingtong He, 2012) Xuan He proposed the assumption of "Moderate Rational Economic Man" based on traditional Chinese ethical ideology. (Xuan He, 2011)

However, the assumptions above have limitations. Nevill Keynes argued that "Assumptions of people's motives or their material and social circumstance do not have to be universally of infinitely correct. Any attempt to make assumptions conform to the so-called 'full empirical experience' is at the expense of generality. As a consequence, we will be drawn into the complexity of realistic economic lives again." (J. Nevill Keynes, 2001) The problem that the models above share is that the conciseness of the rational economic man assumption and the generality it generates are not achieved. That is to say, the behavioral model that gives full consideration to human's social attributes will be too complex to finish mathematical modeling. Therefore, the application scale of it is limited and it is difficult for it to shake the position of the rational economic man assumption in western mainstream economics.

Meanwhile, as the market-oriented economic system reform in China continues to improve, western mainstream economics is gradually taking a leading position and has long been the core curriculum for the bachelor's degree program of economics major students in China's universities. However, the theoretical system of western economics is built under the social background of the Christian culture, and noticeable ethical conflict comes if it is discussed considering eastern culture.

In many specific cases, it will apparently hamper China's efforts to construct legalization. Thus by looking back on the evolution of western mainstream economics methodology, it is necessary for us to analyze in depth the realistic foundation of the rational economic man assumption, and to discuss the ethical conflict to mainstream economics brought by the cultural difference between western and eastern under various social circumstances. Thereupon a new behavioral model of economic man that combines individual rationality and his social attributes can be set up to explain the economic man behavior in realistic economic activities from a new perspective. 


\section{Analysis of the Reality of Rational Economic Man Assumption}

\subsection{Theories Review of Rational Economic Man Assumption}

The assumption of "Rational Economic Man" is from Adam Smith's observation, analysis and summary of the Scottish civil society. Although his following-up economic theories made notable achievements, they were off his original starting point. From The Nature and Causes of the Wealth of Nations and The Theory of Moral Sentiments finished intermittently by Smith, we can summarize Smith's basic principle of economic man behavior. "Smith never indicated that they are only driven by egoism. To be concise, the society cannot build its future on the noblest motive and it should employ the most powerful motive in the most possible way." (Tod. G. Buchholz, 2004)

We should be aware that Adam Smith's classical political economics is originally "a study of wealth as well as a study of man" (Marshall, 2005) and the methodologies he adopted are history inductive approach and abstract deductive approach combined. Thus, in classical political economics, economic man possesses at the same time the individual rationality and sociality. Modern economics has developed into a study that "regards the human behavior as the relation between purpose and rare method that could be employed for various applications", making itself pure "science of rare method configuration". (Lionel Robbins, 2000) Its methodology evolved to a situation where only Jiatu Li's abstract deductive approach survived. Besides, with the development of mainstream economics, abstract deductive approach was improved and it facilitated the mathematical description of rational economic man behavior gradually. Ultimately it developed into Friedman's tool rationalism methodology, which weakens the reality of the assumption and emphasizes theoretical explanation and the ability of prediction. In this field, the assumption of "Rational Economic Man" becomes a reasonable model for analyzing human economic behavior.

However, Adam Smith, the founder of modern mainstream economics, did not agree that the decision-making process of economic man could be so simplified. "Adam Smith is not an egoist who sees self-interest the only driving force of economic activities. The so-called liberalism opinions, including the idea of "Invisible Hand", can only exert their effect under specific conditions. The followings are required: impartial spectator based on sympathy as inward supervision; legal system centering on justice as the basic framework of social functioning; liberal competition under condition of entire fairness." (Patricia Werhane, 2006) This shows that the rationality of the rational economic man assumption is more of the rationality based of mathematical approaches and thus to a certain extent violates Adam Smith's full description of economic man behavior.

According to the "individual process $\leftrightarrow$ group process $\leftrightarrow$ organization process" analytics, Jerrold Greenberg analyzed the mechanism of action an individual adopts when participating in organization activities. He pointed out that there are multiple states of interpersonal relationship, including conflict, cooperation, trust, abnormality and so on. Also he proposed a continuous mapping of types of interpersonal relationship, (Jerald Greenburg, 2011) which contains two extreme states: one is the extremity of unconditional conflict and the other is the extremity of unconditional cooperation. Both are extreme states in theory and therefore their stable existence in reality will be impossible.

Due to the fact that the fundamental definitions of the rational economic man assumption consist of "the selfishness assumption" and "the maximization principle" of human, not a single social condition is included in the definitions. Thus, if we put rational economic man into Greenberg's mapping and analyze it, we can see that rational economic man is in a condition of unconditionally confronting the extreme social relationships. That is to say, rational economic man is inevitably at all times confronting other individuals under the social circumstance. Apparently, rational economic man will fail to survive without an ideal market mechanism, which is a harsh term to achieve. Thereupon, the full assumption of rational economic man implies without question the condition of an ideal market mechanism.

Focusing on the lack of sociality of the rational economic man assumption, many scholars, including scholars in economic circles, proposed various behavioral models of economic man. Nevertheless, as it is discussed above, although these models are more realizable than the rational economic man assumption, it is extremely difficult to simplify them when it comes to mathematical modeling because of the increase of kernel variables concerning economic man's decision-making process.

We can see that the unreality is the advantage and at the same time the disadvantage of the rational economic man assumption, which on one hand extremely simplifies the mathematical modeling and theoretical analysis of economic man behavior and on the other hand extremely limits the application range of the assumption. As a consequence, the assumption can only be applied to the analysis of some economic issues concerning technology alone under specific social circumstance. In western society, the extreme behavioral model that neglects human's 
social attributes can bring rather minor negative effect and the effect can be ignored. To exemplify, the Christian culture that lasts for nearly one millennium has set up social norms that are compatible with the rational economic man assumption. Most importantly, the opinion of the "sin" of human nature in Christian culture has become an essential base point of social norms. Starting from this point, western society not only values the legal norms construction and confines the weakness of human nature as possible, but also in the moral sense counts on the restriction of human weakness from religious fellowship. Under the circumstance of western society, therefore, cooperation between strangers becomes natural on the basis of social norms built by restrictions of law and ethic. The most authorized interpretation of this issue is Weber's thesis: "Market economy in a society dominated by interpersonal relationships is a common case while capitalist market economy in a society ruled by law instead of human relationships is a special case. Weber said, pure capitalism is not seen outside western society because Christian traditions last for nearly a millennium in Western Europe and Western Europe undergone harsh puritan training like Calvinism.”(Dingding Wang, 2011)

It can be seen that the achievements of western mainstream economics not only lie in the perfection of its theoretic system but also its fitness with the social circumstance of western Christian culture. Rigorous ethical restrictions of Christian can cover the inherent defect of the rational economic man assumption, whose conciseness can provide rather convenient approach for the establishment of the following-up economic theoretic system. And that is why the rational economic man assumption exists as an irreplaceable role although its defect looks quite noticeable.

\section{Proposal of Information Economic Man Assumption and Analysis of Its Rationality}

\subsection{Proposal of Information Economic Man Assumption}

Facing multiple doubts and challenges, western mainstream economics made continuous rectification of the connotation of rational economic man assumption. But the rectification mainly focused on peripheral conditions such as "rationality" and "information". In Simon's opinion, "rationality is limited. Economic man, in fact, achieves satisfying degree of efficiency or profit instead of the optimal ones." (Herbert A., 1998) He further proposed the concept of "bounded rational economic man". Meanwhile, the market environment that bounded rational economic man faces is incomplete in information, making the information between economic man's asymmetric. By correcting the periphery conditions of rational economic man assumption, the continuous development of western mainstream economics is promoted and new research areas such as information economics as well as evolutionary economics emerge.

The core assumption of rational economic man regards the nature of self-interest as the only rule in economic decisions, which is criticized and rectified by Kahneman, Smith, Sen and so on. Among them, Amartya Sen emphasizes the effect of factors such as value, ethic and sensibility in social choice by employing the concept of "information expansion". Main methods of "information expansion" contain man's effect comparison and direct introduction of standard of value information. By interpersonal comparison, sensitiveness of welfare and inequality of opportunity can be maintained and the importance of fairness as a social ethic in economic decisions can be emphasized. "To make a reasonable judgment on social welfare, the last thing we can ignore is the interest of groups with comparatively weaker power." (Amartya Sen, 2006) The introduction of the standard of behavior value information brings ethics into economic decisions more directly, offering an available theoretic approach to keep a balance between individual desires and social values. "The consciousness of justice is one of the motives that are able and are often able to stimulate people. Social values can play - and are playing an important part in guaranteeing the success of social organizations in multiple forms. These social organizations contain: market mechanism, democratic politics, fundamental civil power and political power, supply of public goods as well as institutions and systems for public action and protest." (Amartya Sen, 2012) So far, however, these efforts haven't formed a rather mature system yet and have exerted rather limited influence on mainstream economics. The dominant reason is that these rectification principles are still waiting for an abstract summarization using a concise mathematical model, which greatly limits their influence.

To solve this mathematical modeling obstacle and as we can see that many defects will occur if we build a model of rational economic man assumption on the confrontation side of human behavior, it should be a reasonable attempt to build a new one on the cooperation side. With that being said, the model of economic man behavior assumption based on this idea is the very basic concept of "information economic man assumption" proposed by this thesis.

The assumption of information economic man incorporates human's social attributes (sociality) into the model of economic man behavior, constituting main variables for economic man behavior decisions together with human's natural attributes (individual rationality). Here, the value orientation of an individual can be used to describe the 
difference between various individuals. That is to say, the value orientation of "rational social man" is similar to that of the society. They are not identical and the difference between them is in a reasonable scale. And it is the introduction of the value orientation difference that enables "rational social man" to describe all states in the whole mapping of human behavior. Two extremities including unconditional cooperation (individuals with identical value orientation) and extreme conflict (individuals with opposite value orientation) can even be achieved.

Then why is this "rational social man" called information economic man? There are two reasons: firstly, the terminology "rational social man" has been adopted by some scholars and the definition is fairly different from that of this thesis; secondly, the theory of economic man behavioral decisions proposed by this thesis uses information field as its major analytic method and the model mainly applies to the analysis of economic behaviors. Thus, the model of "rational social man" is named the model of "information economic man" assumption.

\subsection{Analysis of Rationality of Information Economic Man Assumption}

Modern economic society is an ultra-organization with high degree of labor division and close cooperation, and the latter has become a basic behavioral characteristic of a civilized society. Meanwhile, social norms including legal system, ethic and culture has supported and guaranteed social cooperation significantly.

Information economic man assumption is a model of human's behavior hypothesis starting from the cooperation side. This method of choosing an extreme state as the foundation of the model is an effective way to build a concise model, which is similar to the rational economic man assumption. However, the rational economic man assumption is so extreme that the path it offers is out of reach. Information economic man assumption, on the contrary, is simplified and flexible enough. It provides an access to describe the pedigree of the whole human behaviors from a comparatively simplified assumption. Besides, this method adapts itself to the specialty of eastern relation society well in particular.

Whether human society can maintain and develop depends on the extensive cooperation between people. And the conflict of interest needs to be constantly reconciled and settled. Knight pointed out that the level of civilization a group ultimately achieves depends on how largely they can tolerate individual differences among themselves and can still maintain their cooperation. (Dingding Wang, 2011) Thus we can see that there are different social norms in different societies, and all social norms exert considerable influence on human's economic activities. Difference between eastern and western culture apparently lies in their perception of human nature and the means they adopt to deal with interest issues.

Eastern culture emphasizes diversity and tolerance. For instance, in Chinese cultures and traditions, Confucianism, Buddhism, Taoism seek common points while reserving difference. Each of them has unique influence on Chinese culture, which is greatly different from western culture dominated by Christian culture.

When the economic rise occurred in Japan in the1950s and 1960s and the Newly Industrial Economics soared in the 1970s, western market-oriented economy theory and advanced management theory. However, these economies did not simply copy western market-oriented economy system. On the contrary, they creatively rectified some western economic system and methods with their own unique eastern cultural background respectively considered. Nevertheless, no economic theory with eastern cultural background was created during this process, not to mention the hypothetical theory of economic man behavior model.

Therefore, the information economic man assumption starts from the most fundamental economic theories and seeks for the theory of economic behavior decisions with eastern cultural background. By this means, the rationality of rational economic man assumption concerning methodology is maintained and the specialty of eastern culture is considered, which contributes to the assumption's realistic basis to a certain extent.

\section{Principle of Information Economic Man Behavioral Decisions and Analytic Model}

From the analysis above we can see that in an unsound market, overemphasis of individual rationality fails to make up for the existing system deficiencies in China and even worse triggers ethical crisis. The rational economic man assumption, which is rooted in western culture, highlights the value of individualism that contradicts with the value of collectivism advocated by eastern Confucianism culture. What's more, it does not fully accord with China's efforts to build a system of harmonious socialist value. Therefore, it is necessary to pursue a kind of theoretical innovation. On the basis of the coordination between individual rationality and sociality, we should regard individual sociality as our basic point and incorporate factors such as ethic and culture into the theoretical analysis of economic activities in order to ultimately achieve our aim of explaining realistic social economic activities as well as conforming to eastern Confucianism culture. 


\subsection{Selection of Kernel Variable of Information Economic Man}

A man in a realistic society is a complex creature with diverse benefits targets and some of the targets even contradict with others. Information economic man, as an "ideal" behavior model of human being, should be able to achieve the abstraction of the most fundamental characteristics of human being without being too complex. With that being said, we can argue that the fundamental characteristic of information economic man is the coordination and unification of sociality and individual rationality. Information economic man possesses both the awareness of active cooperation and the rationality of self-preservation. The target of their behavioral decisions is the co-development of self and society. Although these two fundamental characteristics seem to be contradictory, they are the most realistic portraits of social man. From above, "active cooperation" manifests an individual's pro-social characteristic while "self-preservation" manifests the pursuit of self-interest, the individual rationality and the characteristic of diversity between different individuals. The benefits target of an individual cannot be achieved by one's own power but also by the power of society. Concerning this issue, social norms will play an important role. Social norms greatly simplify the procedure and the cost of an individual's behavioral decision, giving access to lower-cost cooperation between individuals and win-win collaboration outcomes with mutual benefits. That is to say, this target could not be achieved by calculating one's own interest and thus the classical economic analytic method of maximizing the effect could not be applied. Instead, we need to borrow Simon's theory of bounded rationality, i.e. the principle of satisfaction to make decisions.

Social norms must be incorporated into the decision model of information economic man. Here, social norms are regarded as the behavioral norms consisting of ethic and culture for a specific society. The formation of social norms can be seen as the result of social development and evolution. "Society is constituted by ethical values, which pass down from generation to generation during the process of socialization. The ethical values are realized specifically by the internalization of norms." (Talcott Parson, 1967) And the behavioral norms of an individual are built on the result of education during one's upbringing. This result is closely connected to the social circumstance where an individual exists. The behavioral norms of an individual should be similar to the social norms but not identical.

Based on the analysis above, capital vector could be adopted as the kernel variable to describe the characteristic of information economic man. Here, the amplitude of capital vector means the size of capital quantity that an economic man possesses. Capital quantity includes physical capital, human capital and social capital and so on, which are all converted into the same unit and therefore could be compared with each other. The direction of capital vector represents the value orientation of an economic man, i.e. the characteristic of his behavioral norms. The capital vector of information economic man represents the ability and the value orientation he possesses in social economic cooperation, and is the kernel variable to describe the characteristic of an economic man's behavior.

It is assumed that individuals in social organizations possess basically consistent behavioral norms with certain difference and the shared part is called organizational norms. Likewise, organizations that help form the society possess behavioral norms with both certain difference and similarity. The shared part of organizational behavioral norms is called social norms. The selection of this fundamental variable offers a rather concise path for information economic man to achieve the coordination of sociality and individual rationality, which is a key point. An individual seeks for cooperation opportunity in social interactions by constantly searching for and comparing various information and then he makes decisions according to the principle of satisfaction. Therefore the fundamental principle an "Information Economic Man" observes in social interactions is provided.

\subsection{Fundamental Assumption of Information Economic Man}

Decision behavior of information economic man meets the two fundamental principles below:

(1) Behavioral principle of coordination of individual rationality and sociality

Firstly, information economic man possesses clear pro-social characteristic in economic activities and has a will of active cooperation, which is the manifestation of his sociality. Secondly, information economic man possesses clear need of individual rationality, which should be met in all social economic cooperative activities he participates. Information economic man possess the ability to keep an coordination between sociality and the need of individual rationality in social economic activities, indicating that there is a two-way selection freedom for economic agent and economic organization to decide whether they should cooperate with each other or not. Non-cooperation equals to the withdrawal from the organization. The fact that an economic agent decides to join in an organization will indicate that he has a cooperative attitude in the organization and that he will not only observe organizational norms but will also express the consciousness of active cooperation in economic activities. 
(2) Decision principle of information vector comparison

The main task of the behavioral decision of information economic man is to decide whether he participates in a specific economic organization (economic activities) or not. Information vector (capital vector) is used to comprehensively express the characteristic of individuality and group character that an information economic man possesses. The procedure of his behavioral decision is: information economic man chooses action strategies by collecting and synthesizing the environment information of the society (information sent from other economic men or economic organizations), and compares it with his behavioral norms information (threshold information). And then the action strategies are simplified into the participation in the cooperation or withdrawal from the cooperation.

Based on the fundamental principles above, an economic organizational mathematical model on the ground of information economic man assumption can be built to analyze both individual and organizational economic behaviors.

4.3 Model of Decision Behavior of "Information Economic Man"-Information Field Model (Min Zhang \& Zhenggang Chi 2013)

According to the decision principles above as well as the field theory, a model of decision behavior of "information economic man", i.e. model of information field, can be built.

Suppose: in specific economic activities, capital vectors of economic man is respectively $\vec{q}_{1}, \dot{q}_{2}$, the vector sizes $q_{1}$ and $q_{2}$ respectively represent the capital quantity an economic man possesses, the vector direction represents the value orientation of an economic man. So the force of information field between two economic men is:

$$
\vec{F}_{12}=k \frac{q_{1} \cdot q_{2}}{r_{12}^{2}} \cdot \cos \alpha \cdot \vec{e}_{r 12}
$$

In the formula, $k$ is information field constant, is affected by the circumstance where an economic man exists (reflects the efficiency of release and propagation of information). $r_{12}$ is the information distance between economic men (reflects the change of information propagation as the position changes between economic men), $\alpha$ is the space angle of capital vector of two economic men, $0^{\circ} \leq \alpha \leq 180^{\circ}, \vec{e}_{r 12}$ is the direction of field force, and a unit vector that works in the connector of two economic men. Its nature depends of the space angle relation of economic man's capital vector. $\cos \alpha$ 's notation determines $\vec{e}_{r \mid 2}$ 's direction, and when $\cos \alpha$ is positive, $\vec{e}_{r 12}$ points to the other side and the nature of field force is attractive force; when $\cos \alpha$ is negative, $\vec{e}_{r 12}$ is opposite to the other side and the nature of field force is repulsive force, i.e. separating force.

The model above describes that every economic agent or economic organization will set up their own cooperative information fields centering on themselves. Interactional force of information field exists in two economic men (economic organizations) in the space. Its size is proportional to the product of their capital quantity and inversely proportional to the square of their information distance and is connected to the degree of their value orientation difference. The nature of the force is determined by the degree of similarities and differences of their value orientation. If their value orientation is basically the same, the force between them is attractive force, i.e. cooperative force; if basically opposite, the force is repulsive force, i.e. separating force. Picture 1 offers the space schematic diagram of capital vector $\vec{q}_{1}, \dot{q}_{2}$.

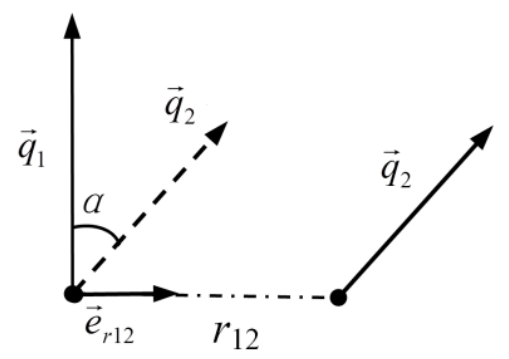

Figure 1. Capital vector space schematic diagram of economic man

According to formula (1), it can be seen in picture 1 that on the premise of unchanged economic condition, when two different economic men are cooperating, the information field force is bigger as the convergence degree of 
participators' value orientation in economic activities is higher. Otherwise, the information field force will be smaller. When the degree of difference of value orientation achieves a certain level, the information field force can be so small and can even be negative that the cooperation cannot be realized. In direct cooperative relation between economic men, only when $0^{\circ} \leq \alpha<90^{\circ}$, the information field force can be positive and the cooperation can be possible. And when $\alpha=180^{\circ}$, the two sides are in a state of absolute conflict and confrontation.

However, whether the cooperation can be truly realized depends on the comparison results of information field force and cooperation threshold between economic men. Only when the information field force between economic men (or economic organizations) is positive, and the force (cooperative force) is bigger than the participators' cooperation thresholds respectively, substantive cooperation occurs. Therefore, the condition for cooperation between information economic men is:

$$
F_{12}-F_{i \cdot s e t} \geq 0, i=1,2,
$$

In the formula, $F_{i \cdot s e t}, i=1,2$, and equals to the cooperation threshold of economic man.

\subsection{Analytic Method of Cooperation Benefit of Information Economic Man}

We need to emphasize that the value orientation difference of economic men and the interest demand difference are two different concepts. The difference of value orientation represents the degree of similarities and differences in terms of social behavioral norms of economic men (economic organizations). In the model of this thesis, $\cos \alpha$ represents this factor's influence on the cooperation and therefore directly incorporates the ethic and culture factors into the model of economic behaviors. And the difference of interest demand is commonly seen in economic cooperation. In this model, economic man's own interest demand is represented by his cooperation threshold. Regarding different economic cooperation, economic man possesses different cooperation threshold accordingly. Therefore, different cooperation threshold represents different interest demand.

For the benefit analysis of economic man in cooperation, the excess benefit that an economic man gains can be represented by the difference between cooperative force and his cooperation threshold. And KR represents the relative value of excess profit, i.e. the excess profit rate. As is in formula (3):

$$
K R_{i}=\frac{F_{12}-F_{i \cdot s e t}}{F_{i \cdot s e t}}, i=1,2,
$$

In formula (3), cooperation threshold represents economic man's expectation of the minimum level of cooperation benefits in the form of cooperative force. From the perspective of benefits, the premise of cooperation is that the excess profit rate of all participators is non-negative. Even when the excess profit rate is null, as long as the cooperation is conducted, economic man's most fundamental benefit is guaranteed. Apparently, with a certain cooperation threshold of economic man, the bigger is the cooperative force, the more benefit economic man can gain in this cooperation and the more stable is the cooperation. The excess profit rate gives a subjective judgment for economic man's benefit in the form of relative value. In the model of information field, the information field force in economic cooperation is the same size to the two cooperative sides but there can be a great difference of their cooperation thresholds respectively. Therefore, the excess profit rate of the two sides could be different in the same cooperation.

From the perspective of the integrity of economic cooperative activities, every economic man faces the issue of distributing the cooperation benefits. The principle of "fair distribution" under the market mechanism distributes benefits according to the proportion relation of the capital quantity each participator invests in. The more is the capital quantity, the more is the benefit ultimately. In the model of information field, capital quantity has a direction. Therefore, the capital quantity needs to be converted by value orientation and then the proportion of benefit distribution can be decided according to the size of capital quantity that is truly invested into the cooperation. This is an objective distribution principle of benefits that can be applied to deal with the distribution issue of excess profits in cooperation.

\section{Conclusion}

Human being makes an individual decision under a specific social ethical circumstance, so do enterprises. It is not only unnecessary but also impossible to neglect an individual's social attributes in these decisions. It is these social attributes of social value information that help people to make relevant decisions in a rather simple manner instead of making action strategies by complex and even unsolved games and calculations. Amartya Sen adopted the method of information expansion to add essential ethical factors to the behavioral model of economic man, which is the basic starting point of the "information economic man" assumption. And the model of information 
field fully expresses the rationality of the principle of fairness as well as the importance of ethical factors in economic decisions. It can be said that the information field model the thesis proposed conforms to the basic law of social selection proposed by Amartya Sen.

Whether the power of social justice is strong or not depends on the level of acceptability of the common social value orientation. Both the power of law and the power of moral are established on the ground of social acceptability. Only when the power of social justice is widely accepted in the society can it truly play its role. Facing the current situation that the social value orientation is developing diversely, we must inspect carefully whether each diversified branch contains the core idea of common social value orientation in order to set up a system of social common value, i.e. the core value system. Or if the branch overemphasizes conflict and confrontation and the anti-society idea becomes a mainstream one in diversified cultures in the society, the core value system can hardly be built. From this perspective, the innovation of economic theories concerning the construction of social common value orientation is particularly significant.

Economic cooperation between human beings is essential to the social developments while there are benefits conflicts between individuals. Therefore, both successful and unsuccessful cooperation existed. And then how can we explain this by using economic theories? The explanation from mainstream economic is excessively pessimistic, regarding conflict as human nature and a failed cooperation is normal. It also deems that a successful cooperation can be achieved only under a rather harsh condition. However, the realistic society is not so negative. The explanation of mainstream economics did not really play a positive role in building a common value system that forms social cooperation. The theory of information field based on information economic man assumption gives a direct and rational explanation on human beings' economic cooperative behaviors, indicating that cooperation is normal and is a target that an individual pursues. Unsuccessful cooperation happens because of lack of social coordinative ability (information communication) and is abnormal. This explanation is not only close to the realistic society but also is helpful to built common social value orientation. Therefore, this innovative study hopes to make some certain corrections on the basis of previous studies. Hopefully, the efforts that this study made is valuable and can enrich as well as improve the decision theory of economic behaviors to some extent.

\section{Acknowledgments}

The authors would like to express heartfelt thanks to the student Zhu zhiyi for her helpful discussions. We also appreciate the Huizhou University for providing financial support.

\section{References}

Amartya Sen. (2006). Rationality and Freedom, P. 67. China Renmin University Press.

Amartya Sen. (2012). Development as Freedom, P. 261. China Renmin University Press.

Daniel A. Wren. (2000). Evolution of Management Ideas, P. 480. Beijing: China Social Sciences Press.

Deqing Wang. (2004). Managerial Psychology, P. 86-88. Chongqing University Press.

Deshun Zang. (2009). From Karl Polanyi to Mark Granovetter: The Backwash and Transcendence of the Assumption of Economic Man to Social Man. Journal of Gansu Institute of Public Administration, 6, $11-20$.

Dingding Wang. (2011). Behavioral Economics Handouts, P. 75. Shanghai People's Publishing House.

Dingding Wang. (2011). From Philosophy to Economics. New Century, 28, 102-103.

Frank Goble. (1987). The Third Force: the Psychology of Abraham Maslow, P. 42. Shanghai Translation Publishing House.

Herbert A. Simon. (1998). Administrative Behavior, P. 18. Beijing College of Economics Press.

Herbert Gintis. (2011). The Bounds of Reason: Game Theory and the Unification of the Behavioral Sciences, P. 172. Shanghai People's Press.

J. Nevill Keynes. (2001). The Scope and Method of Political Economics, PP. 89-150. Huaxia Publishing House. Jerald Greenburg. (2011). Organizational Behavior ( $9^{\text {th }}$ ed.), P. 396. China Renmin University Press.

Jingtong He. (2012). The Limitations of Homo Economicus of Neo-classical Economics and Its Reconstruction. Academic Monthly, 4, 73-80.

Lionel Robbins. (2000). An Essay on the Nature and Significance of Economic Science, P. 19-20, 24. Commercial Press. 
Marshall. (2005). The Principles of Economics, P. 23. Commercial Press.

Min Zhang, \& Zhenggang Chi. (2013). The Study of the Supply Chain Cooperation Mechanism Based on the "Information Economic Man Assumption". American Journal of Industrial and Business Management, 3(4), 418-428

Patricia Werhane. (2006). Adam Smith and His Legacy for Modern Capitalist, P. 1. Shanghai Translation Publishing House.

Shuguang Zhang. (1999). System.Subject.Behavior, P. 64. Chinese Financial \& Economic Publishing House.

Talcott Parson. (1967). Sociological Theory and Modern Society, P. 56. New York: Free Press.

Tod. G. Buchholz. (2004). New Ideas from Dead Economists, P. 20. China Social Sciences Press.

Xinjian Feng. (2001). World-famous Masterpieces of Management Science, P. 59-70. Beijing: Business Management Press.

Xuan He. (2011). Seek for Logical New Starting Point of Rational Economic Behavior. Journal of Finance and Economics, 5, 58-67.

Yaojun Sun. (1999). History of Management Ideas, P. 245. Taiyuan: Shanxi Economics Press.

\section{Copyrights}

Copyright for this article is retained by the author(s), with first publication rights granted to the journal.

This is an open-access article distributed under the terms and conditions of the Creative Commons Attribution license (http://creativecommons.org/licenses/by/4.0/). 